\title{
La diffusion de la publicité par les technologies de l'information et de la communication
}

\begin{abstract}
Résumé:
Information communication technology, constitute a media for as well as a new means which enhanced the filed of advertisement considerably in addition to that, It is an interactive form of communication which allows people dealing in commerce and industry to improve the sell of their products.

Advertisement on the net represents the commercial basis of revenue of sponsors and of the contents and of the different means of research. it gives internet users accesses to sources of info and meant for free which is only possible thought his means .That is advertisement messages are today transmitted through internet in parallel with the traditional means communication.

The right of advertisement, juridical arrangements and applicable legislation should cope with the change on economics, in life style, development in advertising techniques and new materials, like new information technologies and communication.
\end{abstract}

Mots clés : Publicité commerciale ; NTIC ; Internet ; communication commerciale ;

\section{BELIMANE Yamina,}

Faculté de droit

Université des Frères

Mentouri Constantine

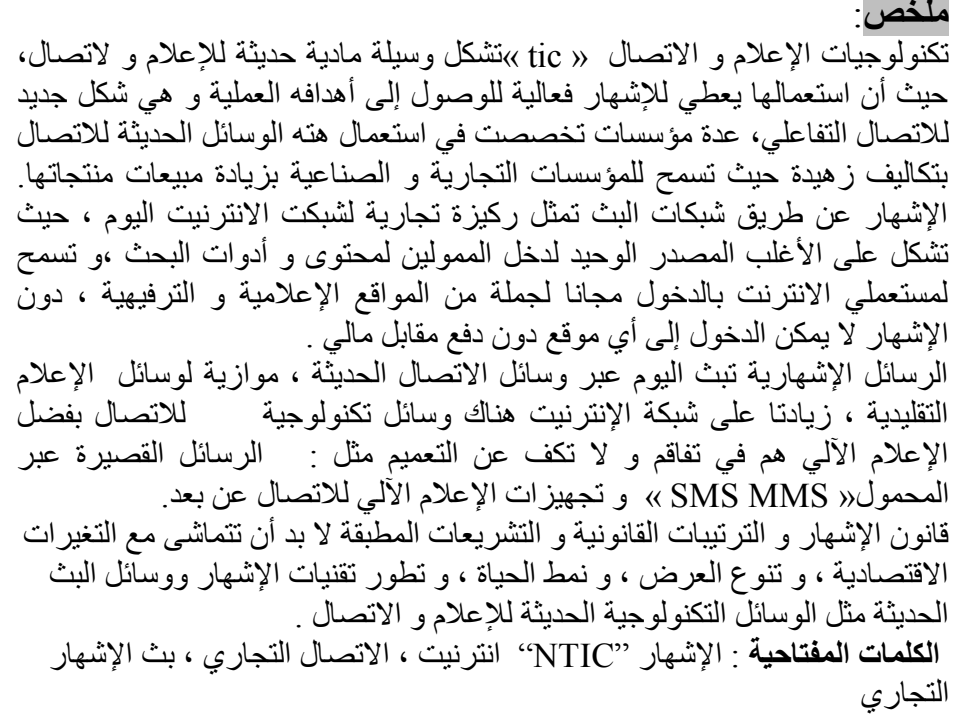

(c) Université des Frère Mentouri Constantine, Algérie, 2016.

\section{Introduction :}

Les technologies de l'information et de la communication (TIC) sont des techniques utilisées dans le traitement et la transmission des informations, particulièrement dans le domaine de l'informatique, de l'Internet et des télécommunications. Ces TIC constituent un média et un nouveau support, dont les usages apportent à la publicité une efficacité dans l'atteinte de ses objectifs opérationnels. C'est aussi une nouvelle forme de communication qui est interactive. \section{.} 年 . 
Les technologies de l'information et de la communication (TIC) sont des techniques utilisées dans le traitement et la transmission des informations, particulièrement dans le domaine de l'informatique, de l'Internet et des télécommunications. Ces TIC constituent un média et un nouveau support, dont les usages apportent à la publicité une efficacité dans l'atteinte de ses objectifs opérationnels. C'est aussi une nouvelle forme de communication qui est interactive.

La publicité a besoin simultanément de capter l'attention, de la retenir et de faire passer son message. Pour atteindre ces objectifs elle doit utiliser les nouvelles technologies d'information et de communication qui sont en pleine croissance et en émergence sans précédent, qui contribuent à une meilleure circulation de l'information à l'échelle planétaire.

Les supports traditionnels, qui sont aussi les vecteurs à l'origine de la construction de la publicité, sont: les affiches, la presse, la radio, puis la télévision. Aujourd'hui ces supports changent et la difficulté pour la publicité est de savoir anticiper ces évolutions, parce qu'avec leur développement, Internet et les nouveaux réseaux sont maintenant pour la publicité des créneaux incontournables pour atteindre tout le monde, et aussi cibler chacun en fonction de ses caractéristiques personnelles. Aujourd'hui le commerce à distance est de retour grâce à l'Internet, qu'on appelle le commerce électronique ou «ecommerce ». Le temps du message visuel, adressé uniformément à tous, est révolu. Une économie du commerce en ligne voit le jour, faisant naître une nouvelle forme de publicité, passant par une approche systématique de plusieurs vecteurs à coordonner. La publicité en ligne doit respecter les règles générales applicables à l'activité publicitaire

\section{1- la publicité commerciale et la loi}

La publicité est le domaine le plus vulgarisé, du moins dans sa forme, par rapport à tous les moyens de communication. L'activité publicitaire est devenue un phénomène qui se développe. Au début, la publicité se trouvait dans sa forme la plus fruste et s'appelait «réclame»; depuis la fin de la seconde guerre mondiale, elle ne cesse de se généraliser et de s'universaliser. Selon le premier dictionnaire de l'Académie française, en 1694, publicité se dit «d'un crime commis à la vue de tous». Le sens du mot publicité en français, signifie étymologiquement « rendre public ». ${ }^{(1)}$ En anglais, publicité se dit « advertisement» ou «publicity», en arabe 《إنهار 》. La «pub» est l'apocope du terme publicité, surtout dans le milieu de la communication et du commerce.

Dans son sens le plus courant, la publicité est «le fait d'exercer une action sur le public à des fins commerciales; le fait de faire connaitre (un produit, un type de produit) et d'inciter à l'acquérir $\aleph^{(2)}$. Elle est considérée comme une forme de communication communément liée aux activités 
commerciales, dont le but est de fixer l'attention de sa cible, le consommateur, de faire connaître une marque, inciter le public à acheter un produit, à utiliser un service. Pour inciter les usagers, utilisateurs, électeurs, à adopter un comportement souhaité, la publicité peut viser la promotion de valeurs considérées comme positives ou bénéfiques pour toute la société. L'intérêt primordial pour les professionnels est donc l'utilisation de ce moyen pour faire connaître leurs produits et leurs services. La publicité constitue sans aucun doute le premier moyen de promotion des ventes et le plus efficace. Mais elle n'est pas sans intérêt pour les consommateurs : elle leur permet de connaître les produits ou services; l'information est attrayante ; la publicité à la télévision principalement, quelquefois considérée comme un véritable spectacle, est très regardée notamment par le jeune public. ${ }^{(3)}$

Elle s'impose aux personnes, il est difficile de l'éviter. Par la séduction, elle exerce son ancrage dans la culture des gens et dans leurs pratiques quotidiennes ; par sa proximité avec les consommateurs, elle devient une partie intégrante de la société dans laquelle elle évolue. Elle est sur la place publique. Elle ne cesse de se développer, d'occuper de nouveaux espaces. Au cours des dernières années, le volume des investissements publicitaires a plus que doublé. L'objectif premier de la publicité est commercial, elle est d'ailleurs considérée comme l'outil majeur de la communication commerciale qui est payante, l'annonceur devait payer pour envoyer son message. Elle est unilatérale, à sens unique de l'annonceur vers la cible visée, mais aussi impersonnelle contrairement à la vente par l'intermédiaire de représentants. La publicité ne s'adresse pas à un individu mais à l'ensemble de ceux qui constituent sa cible.

\section{1-1 la publicité n'est pas exclusivement marchande}

Pourquoi faire de la publicité ? : Faire de la publicité, en général, c'est essayer de capter l'attention du consommateur dans l'objectif de le persuader à acheter. Dans un autre sens, la publicité peut être lancée par les organismes à but non lucratif; dans ce cas, le terme "commercial" est remplacé par "utilitaire" qui supprime la notion d'argent. Il existe aussi des publicités collectives réalisées pour le compte d'une profession, d'un ensemble de producteurs, etc. Puis elle s'est développée pour s'étendre à d'autres activités, entre autres, l'élection des personnalités politiques, la préservation de la nature ou l'incitation à l'économie d'énergie, la mise en garde contre les drogues, ou encore la promotion de la prévention routière, d'un lieu touristique, d'une organisation gouvernementale ainsi que des événements sportifs ou culturels, etc.

Publicité pour qui ? : Elle n'est pas destinée uniquement au grand public, acheteur de biens et de services de consommation courante. Mais peut aussi s'adresser à l'acheteur professionnel, par exemple en milieu industriel et médical.

Comment se fait la publicité ?: Elle s'exprime de deux manières : soit les messages publicitaires sont en mode dénotatif c'est-à-dire qu'ils informent, 
s'adressent à la raison, argumentent pour convaincre, en utilisant le texte, le dessin technique ou l'image, pour renforcer l'explication verbale. Soit en mode connotatif qui évoque et suggère, elle s'adresse à l'émotion, à l'affectivité, aux motivations inconscientes ; l'image est son vecteur privilégié. ${ }^{(4)}$

\section{1-2 La publicité, principal mode de communication commerciale}

La publicité est l'un des aspects de la communication commerciale, c'est-àdire qu'elle fait partie des quatre éléments d'action sur le marché ou marketingmix (le produit, le prix, la distribution, la communication). A ce titre, elle doit informer, certes (sur l'existence du produit, sur son prix, sur ses dimensions...), mais surtout inciter à l'achat, car c'est là sa fonction première. C'est pour atteindre cet objectif que la publicité cherche comment toucher, puis convaincre (séduire) l'acheteur potentiel. Cette recherche a évolué depuis cinquante ans pour aboutir à la synthèse actuelle dont et à travers laquelle, "pour le conduire à l'achat, la publicité présente au consommateur, à des fins d'identification, une image de lui-même conforme à ses attentes; celle-ci étant fonction de l'environnement socioculturel et par là même normative, elle doit, en première analyse, $s$ 'y conformer $\gg .{ }^{(5)}$

La publicité représente une forme importante de la communication et comme toute communication, quelle que soit sa forme et quel que soit son objet, commercial ou non, elle fonctionne comme un système reliant un émetteur et un ou plusieurs récepteurs. Les professionnels de la publicité travaillent sans relâche pour faire évoluer ses méthodes et ses techniques, qui se concrétisent dans les messages insérés dans les grands médias: presse, télévision, affichage, radio, cinéma et maintenant Internet.

Dans sa manifestation la plus concrète, la publicité apparaît comme la réunion d'arguments, d'informations et de moyens de persuasion tendus vers un but unique : la vente. C'est le fait d'exercer une sollicitation psychologique sur le public à des fins commerciales. C'est la finalité de la publicité commerciale, son but lucratif, qui détermine sa différence et la distingue de l'information pure et de la propagande, même si avec cette dernière, elle a quelques points communs, comme le rôle d'exercer une influence sur les personnes et les groupes auxquels elle s'adresse, et aussi pour ce qui est des techniques utilisées. La propagande est au service de la diffusion d'une idéologie, elle met en valeur des opinions ou une doctrine, elle n'a en aucun cas de préoccupation commerciale.

\section{1-3 La publicité et autres notions}

Un autre critère nous permet également de distinguer la publicité des relations publiques : dans une entreprise, par exemple, le service des relations publiques a pour fonction d'assurer des communications pas nécessairement commerciales, plus harmonieuses entre cette même entreprise et son environnement. Les relations publiques créent une atmosphère de sympathie, 
comme pour développer l'idée de la corrélation entre l'économie, l'environnement et le social.

Les entreprises et leurs marques doivent s'investir en pratique, par le marketing et la publicité de valeurs sociales nouvelles qui se résume dans trois domaines qu'il faut rendre compatibles : l'environnement, l'économie et le social. De fait, quelques entreprises annoncent des engagements concrets dans leur communication commerciale en faveur de la cité et du développement durable, comme c'est le cas des grandes marques qui appartiennent à des sociétés multinationales.

La publicité « insulte nos regards, falsifie les épithètes, gâte les paysages, corrompt toute qualité et toute critique » selon le célèbre écrivain français Paul Valéry.

Par contre d'autres auteurs disent avec Blaise Cendrars que la publicité est «La fleur de la vie contemporaine, une affirmation d'optimisme et de gaîté ». L'omniprésence de la publicité dans la vie quotidienne des gens, les fait souvent rêver.

Bien que ces propos ne reflètent pas la stricte réalité, ils contiennent une part de vérité. D'autres spécialistes comme les sociologues ont analysé plus froidement le phénomène de la publicité. Selon une étude réalisée par d'éminents professeurs de quelques universités parisiennes, ont essayé de trouver des réponses dans des situations de paradoxe, et de rechercher l'impact social de la publicité à travers un certains nombre de questions comme : "Le phénomène publicitaire détermine-t-il des attitudes sociales, des modes de pratique et de réflexion sur celle-ci ou bien est-il le reflet des transformations de la vie sociale... » Le résultat de la plupart des recherches sur l'impact social de la publicité, reste insaisissable, d'après les spécialistes en sociologie. La redondance des slogans, par exemple, n'est qu'un facteur secondaire par rapport à la forme de l'échange effectuée par l'émission d'un langage impératif (phases stéréotypées, réponses prédéterminées, propos exhaustifs).

Le phénomène publicitaire agit simultanément sur les modes de comportement social, les formes de l'échange, les structures de la communication sociale, selon un processus d'information progressif. Il influe directement sur l'ensemble des valeurs et des représentations sociales et contribue aux transformations de la vie sociale, du bouleversement des conditions de la production et des formes de la consommation.

La publicité est devenue aujourd'hui un élément culturel qui accompagne la vie autant qu'elle essaye de l'influencer, et elle va même jusqu'à puiser dans nos interrogations existentielles. Mais derrière un slogan percutant, une image soigneusement travaillée, un spot à la télévision ou à la radio, se cachent toute une industrie et des professionnels.

1-4 Les praticiens de la publicité commerciale et son encadrement juridique 
Selon J.J. ROUSSEAU : «La loi qu'on se donne librement s'appelle liberté, à condition de l'écrire en pleine autonomie. »

Certes les professionnels responsables ont besoin de la loi pour que leur liberté d'entreprendre, de créer, de diffuser leurs messages, soit garantie. L'autorégulation et l'autodiscipline complètent aussi la loi, qui à son tour confirme des codes de conduite professionnels, pour éviter d'éventuelles dérives dans cette matière et abuser du consommateur (comme la publicité mensongère ou la publicité trompeuse).

Certains auteurs parlent du droit de la publicité commerciale et souhaitent le codifier, ils le considèrent comme une branche du droit de la communication, bien qu'il soit géré par des textes épars et des disciplines diverses. Les sources du droit de la publicité sont multiples, commençant par les principes fondamentaux du droit des contrats et de la responsabilité civile, et plus particulièrement le droit de la consommation et le droit de l'environnement. Il s'appuie sur le code du commerce et sur le code pénal en cas de non respect des dispositions règlementaires. D'autres textes complètent le cadre juridique de l'activité publicitaire comme le code de la santé publique, le code de la propriété intellectuelle et même le code de la route.

Le droit de la publicité en tant que matière juridique n'existe pas. Pourtant, la publicité lui est soumise. Ainsi, la publicité est assujettie et encadré par le droit afin d'éviter les déviances. S'agissant de savoir comment créer une harmonisation entre le domaine de la publicité, qui est en perpétuelle mutation, et la loi. La genèse de la publicité est le produit de divers facteurs dont le plus important est sans conteste l'enjeu commercial. Tous les espaces de communication commerciale convergent, il n'y a plus de frontière maintenant entre communication et marketing. On est en face d'un nouveau marketing et d'une nouvelle communication fondés sur l'observation et l'analyse des comportements individuels et non seulement sur l'interprétation des opinions, des motivations et des attitudes des consommateurs. Cette situation rend la concurrence plus accrue et ouvre la voie à tout type de dérive.

Concernant le droit algérien, les insuffisances d'un encadrement juridique en matière de publicité commerciale sont évidentes à l'image de la loi $\mathrm{n}^{\circ}$ 04-02 du 23 juin 2004 fixant les pratiques commerciales, qui s'est contenté d'énumérer simplement quelques cas de publicité trompeuse.

Cet agencement de règlementation, ne peut que servir à optimiser un encadrement judicieux de la publicité dans notre pays.

\section{2 - La publicité par les technologies de l'information et de la communication.}

Les nouvelles technologies d'information et de communication sont devenues aujourd'hui des supports sur lesquels va s'installer la publicité pour atteindre le consommateur, ce qui leur a valu le statut de média. Des sociétés se sont spécialisées pour utiliser ces nouveaux moyens de communication à coût très 
réduit et permettre à des entreprises commerciales ou industrielles d'accroître la vente de leurs produits.

La publicité en ligne représente le pilier commercial du réseau Internet d'aujourd'hui. Elle constitue souvent l'unique source de revenus des fournisseurs de contenu et des outils de recherche. La publicité se retrouve partout sur Internet, notamment sur le Web et dans les contenus multimédias diffusés en temps réel. Elle permet ainsi aux internautes d'accéder gratuitement à une mine d'informations et de divertissements. Sans elle, l'accès aux contenus serait inévitablement précédé d'une contrepartie pécuniaire. ${ }^{(6)}$

Le commerçant électronique désireux d'annoncer ses produits et services, bénéficie d'une ouverture considérable sur Internet. Il peut utiliser ce nouveau média tant pour faire valoir son offre que pour accroître sa crédibilité. En cela, la publicité en ligne n'est guère différente de sa forme traditionnelle : elle vise à atteindre efficacement le consommateur et à agir sur ses décisions.

Outre Internet, d'autre moyens technologiques de communication, grâce à l'informatique, sont en expansion et ne cessent de se répande comme : les SMS, MMS, téléphone, terminaux reliés à des réseaux informatiques

\section{2-1 La publicité par téléphone}

La publicité par téléphone utilisée dans les sociétés de consommations, notamment les pays favorables à l'économie de marché, n'est possible qu'après un consentement préalable du consommateur à recevoir des prospections directes par ce moyen. Outre les textes applicables en France comme la loi pour la confiance dans l'économie numérique, l'Autorité de Régulation Professionnelle de la Publicité (ARPP), ex. BVP : Bureau de vérification de la publicité a publié une recommandation relative au marketing téléphonique et notamment le respect par les publicitaires d'un certain nombre de règles déontologiques qui sont les suivantes :

"Les appels téléphoniques auprès des particuliers ne peuvent êtres effectués que pendant les jours ouvrables, à des heures adaptées à l'horaire et au rythme de leur vie privée.

Les enfants ne doivent en aucun cas être l'objet d'une sollicitation commerciale par téléphone ;

- les messages téléphoniques doivent permettre aux correspondants de comprendre dès le début de la conversation l'objet final de la communication téléphonique (simple publicité, préparation à la vente ou prise de commande) ;

- la publicité par téléphone doit être conçue et pratiquée de manière à ne pas être susceptible d'induire les correspondants en erreur, abuser de leur confiance ou exploiter leur manque d'expérience ou de connaissance. Elle doit également proscrire toute exploitation de la superstition ou du sentiment de peur ;

- la publicité par téléphone ne doit en aucun cas prêter à confusion avec une étude de marché, étude d'opinion ou étude documentaire ; 
- la communication téléphonique ne doit d'aucune façon être susceptible de créer une confusion entre l'annonceur - ou sa marque- et ses concurrents- ou leurs marques ;

- l'annonceur doit s'abstenir formellement de tout dénigrement direct ou indirect d'une entreprise ou d'un produit concurrent. »

Dans un proche avenir, il est probable que le téléphone mobile sera le support le plus répandu pour les campagnes publicitaires. Le nouveau marché de la publicité s'annonce sur la base de trois objectifs : numérique, interactif, mobile.

La publicité par les SMS qui sont de petits messages textuels qu'on peut envoyer sur un téléphone portable également appelés « texto » dont la taille est limitée à 140 ou 160 caractères. SMS l'abréviation en anglais de (short message system). Le MMS, l'abréviation de (Multimédia message service), permet d'enrichir le message texte de type SMS d'images, de séquences animées et /ou de son. Les SMS ou MMS permettent d'adresser tout type de message y compris publicitaire.

\section{2-2 La publicité par Internet}

Internet est le plus connu des réseaux qui participent à ce qu'on appelle « les autoroutes de l'information ». Il est considéré comme le " réseau des réseaux » puisque 45000 réseaux participaient à son maillage en 1996. Réservé aux universités, ce mode de communication qui avait au départ un but scientifique, est devenu un moyen international d'échanges. L'accès à ce réseau n'a été ouvert au grand public qu'à partir de 1994. Il renferme le World Wide Web (www) c'est-à-dire la «toile d'araignée mondiale». Constituée par des milliers de serveurs liés entre eux par des liens hypertextes, les utilisateurs accèdent par le moyen de logiciels.

Internet n'a pas de personnalité morale ni de structure administrative qui le représente vis-à-vis des usagers ou des tiers. Néanmoins il existe une structure, l'Internet society (ISOC), une association d'origine américaine à but non lucratif, qui a pour objet d'assurer la promotion et la mise en place, par le biais de groupes de travail, de standards communs à tous les niveaux du réseau. (6)

Par son statut, Internet échappe à tout contrôle centralisé, c'est pourquoi « aucune nation ne pourra se l'approprier, aucune administration n'en assurer la police ${ }^{(7)}$

Toutefois, la réglementation de la publicité ne constitue pas un droit harmonisé et varie par conséquent d'un État à l'autre. La nature transnationale du réseau Internet pose à cet égard une difficulté particulière. Puisque les contenus disponibles sur Internet sont accessibles de par le monde, il s'avère difficile de s'adresser aux consommateurs selon le droit applicable à chacun d'entre eux. 
Pour apprécier l'utilité du référencement, il faut comprendre que la mise en ligne de sites Web n'est guère orchestrée, c'est-à-dire qu'aucune entité civile, étatique ou internationale ne gère le contenu diffusé sur Internet : les sites Web apparaissent et disparaissent sans aucun contrôle et selon le désir de leurs auteurs. Par conséquent, il est rapidement devenu impossible d'indexer l'ensemble des informations et des sites au fur et à mesure de leur publication en ligne.

Internet, ce réseau informatique mondial, a révolutionné depuis quelques années les relations entre les personnes. Il permet à quiconque disposant d'un ordinateur et d'un moyen d'accès, d'effectuer différentes opérations et de participer aux relations que peut créer ce réseau.

A partir d'un serveur installé dans n'importe quel pays, n'importe qui peut proposer des produits ou services sur ce réseau aux différents utilisateurs. Dans le domaine commercial un consommateur peut, d'un pays à l'autre, acheter, vendre, échanger grâce à un site situé sur n'importe quel point dans le monde, ce site étant considéré comme une boutique pour le vendeur. En 1997, son chiffre d'affaires s'élevait à 10 millions de Dollars, et en 2004, à plus de 450 milliards de Dollars.

Les annonceurs et le monde de la publicité peuvent atteindre directement une clientèle à l'échelle planétaire l'internationalisation des messages publicitaires étant maintenant possible grâce à l'Internet qui ouvre de nouveaux horizons à la publicité.

Parmi les avantages de ce support, l'adaptation de la publicité à chaque consommateur, qui peut être individualisée en fonction des renseignements que l'annonceur peut réussir à obtenir à travers la diffusion de la publicité ellemême, en effet, dans cette publicité il existe un fichier qui peut espionner nos comportements sur un site, fichier appelé «cookie». Un deuxième avantage réside dans la détermination de la valeur de l'espace publicitaire grâce aux nombres de fréquentations de la page publicitaire d'un serveur. ${ }^{\left({ }^{(}\right)}$

\section{2-3 Les formes de publicité sur Internet}

La publicité peut être interactive, les messages publicitaires apparaissent en haut et en bas de l'écran, sous forme de bandeaux. Par des liens hypertextes, l'internaute peut cliquer soit pour obtenir des informations sur le produit, le service ou le prestataire concerné soit pour commander le produit. Un bandeau publicitaire ou bannière est une " petite annonce publicitaire comportant une image ou un bref message qui est affiché généralement dans le haut de la page d'accueil d'un site Web, le plus souvent à caractère commercial, et sur laquelle l'internaute est invité à cliquer ». En général, le clic conduit l'internaute au site de l'annonceur où il peut consulter les informations sur le produit ou service offert.

La publicité peut apparaître en plein écran, pour un bref moment très court, comme le site peut lui-même être un espace publicitaire. La manière la plus 
classique, c'est l'envoi des messages publicitaires «mailing », sur des adresses électroniques présélectionnées.

Lorsque l'annonceur dispose, par exemple, de données relatives aux habitudes de consommation de ses destinataires, il peut aussi cibler son marché en fonction des différents produits et services qu'il offre. Il importe toutefois d'indiquer que ce type d'information est susceptible d'être protégé par la loi. Il n'est d'ailleurs pas certain qu'un annonceur pourra légalement acheter ou vendre des adresses de courriers électroniques.

En conséquence, le commerçant électronique qui exerce des activités de vente se rapportant à des aliments ou drogues devra inévitablement s'assurer de la légalité de ses annonces ainsi que de ses transactions avec les internautes.

Afin de s'assurer de la légalité de son contenu publicitaire, le commerçant électronique devrait au minimum vérifier les règles en vigueur dans son propre pays et fournir aux consommateurs toutes les informations nécessaires à la conclusion d'une transaction honnête. L'exercice d'une bonne pratique commerciale est garante d'une réussite durable et prospère. Dans un contexte où il est difficile d'obtenir la confiance des consommateurs, ce principe soustend une politique exigeante à l'égard des relations avec la clientèle.

En général, cette réglementation a pour objet de protéger les consommateurs contre la publicité fausse et trompeuse. Elle peut également viser à protéger les personnes plus crédules comme les enfants ou à tempérer la consommation de produits nocifs pour la santé. ${ }^{(9)}$

En Algérie, le premier texte applicable aux services offert par Internet, quatre ans après sa mise au service du grand public en 1994, a été le décret $\mathrm{n}^{\circ}$ 98-257 du 25 août 1998, définissant les conditions et les modalités de mise en place, modifié par le décret exécutif $\mathrm{n}^{\circ} 200-307$ du 14 octobre 2000. Les providers ou fournisseurs d'accès à Internet exercent leur profession sur la base d'un cahier des charges qui les lient à l'Autorité de régulation (ARPT). Un cahier des charges définissant les conditions et modalités d'établissement et d'exploitation des services Internet mis en place en application de l'article 39 de la loi $\mathrm{n}^{\circ} 2000-03$ du 5 août 2000 fixant les règles relatives à la poste et aux télécommunications. L'autorisation accordée au titulaire doit être mise en œuvre conformément aux dispositions législatives et règlementaires nationales ainsi qu'aux normes internationales en vigueur.

La loi du 5 août 2009 vient fixer les règles particulières relatives à la prévention et à la lutte contre les infractions liées aux technologies de l'information et de la communication. Ni dans cette loi, ni dans les décrets qui la précèdent, il n'y a pas de mentions ni de règles concernant la publicité sur Internet, à part des obligations à l'égard des fournisseurs d'accès à ce dernier.

Les messages publicitaires sont aujourd'hui diffusés par les nouveaux moyens de communications, Internet et le téléphone portable, en parallèle avec les médias classiques. Ils sont en extension constante. Ensemble ils font 
accroître le poids économique du secteur de la publicité. Les différentes formes de messages publicitaires sont l'œuvre des spécialistes dans la création artistique et technique, agencés par d'autres professionnels qui offrent leur service aux différents annonceurs.

Toute cette panoplie de praticiens de la publicité a crucialement besoin de dispositif transparents comme les contrats, voulus par le législateur afin de minimiser les risques de conflit. Même un cadre des plus performants de règlement ne peut seul suffire à instaurer des comportements adéquats en publicité, une réflexion morale devient plus que nécessaire. L'autodiscipline peut jouer ce rôle, établie par les professionnels eux-mêmes, Nétiquette pour les pratiques et les usages sur Internet. Une déontologie, et même une éthique pourquoi pas, serviraient à protéger les consommateurs, surtout les femmes et les plus vulnérables, les enfants, et même les gens les plus âgés.

$\mathrm{Au}$ terme de cette modeste intervention, les premières déductions qu'on peut tirer selon le raisonnement le plus approprié à se sujet, permet concrètement de dire que la publicité a une importance économique et sociale qui n'est plus à démontrer. Elle peut espérer un avenir plus clément grâce à l'extension des moyens de communication qu'offrent les nouvelles technologies de l'information et de la communication, qui lui ouvrent des champs nouveaux qu'elle va investir. Elle ne peut se développer que si elle aspire à se qualifier comme référence, en adoptant une ligne qui lui permet d'être le reflet d'un label de tout produit et service, qu'elle propose aux différents consommateurs. Mais l'activité publicitaire ne peut se développer qu'en respectant les lois de la cité tout autant que les sensibilités du public. Il est primordial de connaitre mieux la publicité à travers des textes qui donnent à la notion de publicité des définitions claires et précises. Ces définitions insistent tantôt sur la finalité d'un message, tantôt sur son contenu, ou encore sur ses destinataires. Ainsi, on peut définir la publicité comme étant un ensemble de moyens destinés à faire connaître une entreprise, un produit... Sa fonction économique consiste à éveiller l'intérêt et à faire naître le désir afin de stimuler la demande. La publicité vise donc à faire accroître les ventes

La publicité existe, sa licéité n'est pas remise en cause ; elle n'est pas non plus affirmée : le droit explique toujours ce qui est interdit, non pas ce qui est permis. Le droit intervient seulement pour éviter les abus, les dangers de la publicité. Les principes de base de toute publicité commerciale, c'est qu'elle doit se conformer aux lois, être décente, loyale et véridique. Quand le message publicitaire contient une comparaison, celle-ci ne doit pas être de nature à induire en erreur le consommateur et doit respecter les principes de la concurrence loyale.

La publicité doit être clairement distinguée comme telle, quelles que soit la forme et le support utilisé, surtout quand le message publicitaire est diffusé dans des médias qui comportent aussi des informations ou des articles 
rédactionnels. La sécurité n'est pas en marge des règles, qui imposent à la publicité de ne pas présenter ou comporter aucune description de pratiques dangereuses ou de situations ou la sécurité n'est pas respectée, particulièrement dans la publicité présentant des enfants ou adolescents ou s'adressant à eux. Elle ne doit pas exploiter la crédulité naturelle des enfants ou le manque d'expérience des adolescents, ne comporter aucune déclaration ou présentation visuelle qui risquerait de leur causer un dommage mental, moral ou physique.

En Algérie, les professionnels n'ont pas encore essayé de prendre en main l'organisation et la gestion de la publicité commerciale par des procédés d'autorégulation. Cependant le peu de textes qui régissent la publicité reste très insuffisant, par exemple, les deux lois relatives aux règles générales de protection du consommateur qui n'ont à aucun moment cité la publicité commerciale, (loi $n^{\circ}$ 89-02 du 07 février 1989 et loi $n^{\circ}$ 09-03 du 25 février 2009). Il est pourtant du ressort du législateur de protéger le consommateur contre les différentes formes de dérive, d'infractions par les messages nuisibles pour la santé publique, l'ordre public ou l'éthique et les mœurs.

\section{Bibliographie}

1-TEYSSIER, Jean-Pierre, Frapper sans heurter : quelle éthique pour la publicité, p. 12.

2- Dans le dictionnaire, Le nouveau Petit Robert, 2008.

3- PICOD, Yves, DAVO, Hélène, Droit de la consommation, p 67.

4 - DAYAN, Armand, La publicité, pp. 7-9.

5- Idem, p. 5.

6- Thierry Piette, André Bertrand. Internet et la loi. Paris, Dalloz, 1997, p.11.

7 - M.MAY, « Cyberspace : enfer et paradis », dans le journal, le monde.

8- LABBE, Éric, Le guide juridique du commerçant électronique, p.123.

9 - LABBE, Eric, op.cit. p.288. 